\title{
Measured versus Estimated Total Liver Volume to Preoperatively Assess the Adequacy of the Future Liver Remnant
}

\author{
Which Method Should We Use?
}

\author{
Dario Ribero, MD, ${ }^{*}$ Marco Amisano, MD,* Francesca Bertuzzo, MD, * Serena Langella, MD, * \\ Roberto Lo Tesoriere, MD, ${ }^{*}$ Alessandro Ferrero, MD, ${ }^{*}$ Daniele Regge, MD, $\dagger$ and Lorenzo Capussotti, MD*
}

\begin{abstract}
Objectives: To determine which method of liver volumetry is more accurate in predicting a safe resection.

Background: Before major or extended hepatectomy, assessment of the future liver remnant (FLR) is crucial to reduce the risk of postoperative hepatic insufficiency. The FLR volume is usually expressed as the ratio of FLR to nontumorous total liver volume (TLV), which can be measured directly by computed tomography (mTLV) or estimated (eTLV) on the basis of correlation existing with the body surface area. To date, these 2 methods have never been compared.

Methods: All consecutive, noncirrhotic patients who underwent resection of 3 or more liver segments between April 2000 and April 2012 and for whom (i) preoperative computed tomographic scans and (ii) body surface area were available entered the study. The mTLV (calculated as TLV - tumor volume) was compared with the eTLV (calculated as $-794.41+1267.28 \times$ body surface area) using volumetric data $\left(\mathrm{cm}^{3}\right)$ and clinical outcome measures (specifically, hepatic insufficiency and 90-day mortality). Definition of hepatic insufficiency was peak postoperative serum total bilirubin level of more than $7 \mathrm{mg} / \mathrm{dL}$ or, in jaundiced patients, an increasing bilirubin level on day 5 or thereafter.
\end{abstract}

Results: Two-hundred forty-three patients who had undergone major ( $\mathrm{n}=$ $135)$ or extended $(n=108)$ hepatectomies met the inclusion criteria. Twentyeight patients $(11.5 \%)$ developed hepatic insufficiency, whereas 7 patients $(2.9 \%)$ died postoperatively. Compared with the eTLV, the mTLV underestimated the liver volume in $60.1 \%$ of the patients $(P<0.01)$. Forty-seven and 73 patients had an inadequate FLR based on mTLV and eTLV, respectively. Portal vein occlusion (PVO) was used in 44 patients. In patients $(n=162)$ in whom both methods did not evidence the need for PVO, postoperative hepatic insufficiency and mortality were $4.9 \%$ and $0.6 \%$, respectively. Conversely, in patients $(n=27)$ in whom the eTLV but not the mTLV evidenced the need for PVO, and thus PVO was not performed, hepatic insufficiency $(22.2 \%$; $P=0.001)$ and mortality $(3.7 \% ; P=\mathrm{ns})$ were higher.

Conclusions: The use of eTLV identifies a subset of patients ( $\sim 11 \%)$ in whom liver volumetry with the mTLV underestimates the risk of hepatic insufficiency.

Keywords: future liver remnant, hepatic insufficiency, liver volumetry, portal vein embolization, portal vein occlusion

(Ann Surg 2013;258:801-807)

W ith advances in surgical technique and perioperative care, the frontiers of liver surgery are extending continuously toward more major or extended liver resections. ${ }^{1-3}$ This aggressive approach,

From the *Department of General Surgery and Surgical Oncology, Ospedale Mauriziano "Umberto I"; and †Department of Radiology, Istituto per la Ricerca e la Cura del Cancro, Candiolo, Turin, Italy.

Disclosure: The authors declare no conflicts of interest.

Reprints: Dario Ribero, MD, Department of General Surgery and Surgical Oncology, Ospedale Mauriziano "Umberto I," Turin, Italy. E-mail: dribero@mauriziano.it.

Copyright (C) 2013 by Lippincott Williams \& Wilkins

ISSN: 0003-4932/13/25805-0801

DOI: $10.1097 /$ SLA.0000000000000213 however, has been associated with increased postoperative complications, in particular hepatic insufficiency, ${ }^{4}$ and mortality rates of $5 \%$ to $8 \%$ have been reported from specialized centers. ${ }^{5-7}$ Because volume and function of the residual liver are intimately associated, it has being recognized that the preoperative determination of the future liver remnant (FLR) volume is critical to ensure that functionally sufficient liver parenchyma remains. Therefore, liver volumetry has become an essential component of surgical planning.

The FLR volume is usually expressed as a ratio of FLR to total liver volume (TLV) that, in most centers, is measured directly by computed tomography (CT). The tumor volume is routinely subtracted from the measured TLV to consider the extent of hepatic replacement by tumor(s) that does not contribute to the functional liver volume. However, this method of calculation has some inherent disadvantages. ${ }^{8}$ To overcome these limitations, a method, defined as standardized volumetry, that estimates the TLV based on the body surface area (BSA) has been proposed ${ }^{8,9}$ and rapidly followed in the clinical practice. ${ }^{7-10}$ Although many studies have investigated the critical residual liver volume associated with the development of hepatic dysfunction or insufficiency, ${ }^{11-14}$ the clinical implications of the method used to measure liver volumes have not been investigated. In particular, a direct comparison between the 2 methods has never been specifically performed. The purpose of this study was to determine which of the 2 methods of liver volumetry, the traditional CT-based one and the standardized one, is more accurate in predicting a safe, major, or extended hepatic resection in noncirrhotic patients.

\section{METHODS}

All consecutive adult patients who underwent major or extended hepatectomies (ie, resection of $\geq 3$ Couinaud segments) at our institution from April 2000 to April 2012 were identified from a prospectively collected hepatobiliary database. All noncirrhotic patients were enrolled into this study, with the only exception of those for whom (i) preoperative CT scan and (ii) anthropometric data (specifically, body weight and height) were not available for review. Standard demographic, clinicopathological, and volumetric data were recorded for all patients. Data collection and analysis were performed according to the institutional guidelines conforming to the ethical standards of the Helsinki Declaration.

Before resection, all patients underwent a standard preoperative workup, including a routine clinical evaluation, determination of the indocyanine green retention rate at 15 minutes, and a contrastenhanced CT scan of the thorax and abdomen. In selected cases, magnetic resonance imaging with liver-specific contrast agents, ${ }^{15}$ magnetic resonance cholangiopancreatography or direct percutaneous or endoscopic cholangiography, ${ }^{16}$ and positron emission tomography completed the evaluation process. All patients scheduled to undergo trisectionectomies or right hepatectomies and those with an anticipated small FLR underwent CT volumetry and a subsequent portal vein occlusion (PVO), consisting of either surgical ligation ${ }^{17}$ or percutaneous embolization (PVE) of the right portal vein with or without 
embolization of segment 4 portal branches, ${ }^{18}$ when the FLR volume was considered inadequate. Threshold volumes for safe hepatectomy, expressed as the FLR to TLV ratio, were more than $25 \%$ in patients with normal liver and more than $30 \%$ in those who had received extensive chemotherapy, in those with prolonged cholestasis, in those with severe fibrosis, or in those scheduled to undergo complex resections. In jaundiced patients, PVE was performed after percutaneous decompression of the biliary tree on the side of the liver to be left in. Surgery was then scheduled if sufficient hypertrophy of the FLR was obtained 4 weeks after the procedure. ${ }^{10}$

For the purpose of this study, for each patient, we determined the FLR volume, the tumor volume, and the TLV all in $\mathrm{cm}^{3}$. The technique of liver volumetry has been previously described. ${ }^{17,18}$ Briefly, on axial CT images obtained during the portal venous phase after intravenous contrast injection, the hepatic total and segmental contours of each slice were traced manually by a hepatobiliary radiologist. The enclosed area was then determined automatically, and the volume was calculated by summing each surface area multiplied by the slice thickness. With the same procedure, the tumor volume was measured in each patient. Half of the segment 4 volume was included in the FLR volume when liver resection was extended to either segment $4 \mathrm{a}$ or $4 \mathrm{~b}$. The FRL volume was then equated to the TLV obtained by 2 different methods as follows. The first method uses individual CT-measured data and expresses the TLV, hereafter referred to as "measured total liver volume" (mTLV), as the actual nontumorous TLV (mTLV $\left[\mathrm{cm}^{3}\right]$ $=$ total CT-measured liver volume $\left[\mathrm{cm}^{3}\right]-$ tumor volume $\left.\left[\mathrm{cm}^{3}\right]\right)$. This is the method that has been routinely used at our institution during the study period. The second method estimates the TLV, hereafter referred to as "estimated total liver volume" (eTLV), using a mathematical formula derived from the linear correlation existing between liver size and BSA (eTLV $\left[\mathrm{cm}^{3}\right]=-794.41+1267.28 \times$ BSA). ${ }^{9}$ BSA was calculated using weight and height according to the Mosteller formula ${ }^{19}:$ BSA $=[\text { weight }(\mathrm{kg}) \times \text { height }(\mathrm{cm}) \div 3600]^{1 / 2}$. These 2 methods were compared using volumetric data in $\mathrm{cm}^{3}$, corresponding ratios expressed as percentages (measured ratio $\left[\mathrm{mR}_{\mathrm{atio}}\right]$ $=$ FLR in $\mathrm{cm}^{3} / \mathrm{mTLV}$ in $\mathrm{cm}^{3} \times 100$; estimated ratio $\left[\mathrm{eR}_{\mathrm{atio}}\right]=$ FLR in $\mathrm{cm}^{3} / \mathrm{eTLV}$ in $\mathrm{cm}^{3} \times 100$ ), and clinical outcome measures, specifically hepatic insufficiency and postoperative mortality, which was defined as any death occurring within 90 days after surgery or within the hospital stay during which the surgery was performed. Postoperative morbidity and mortality were reviewed on the basis of the classification of Dindo et al. ${ }^{20}$ Complications of grade III or higher were defined as major morbidity. Hepatic insufficiency was defined as a peak postoperative serum total bilirubin level of more than 7.0 $\mathrm{mg} / \mathrm{dL}^{4}$ or, in jaundiced patients, an increasing serum bilirubin concentration on postoperative day 5 or thereafter compared with the values of the previous days. ${ }^{21}$ Hepatic insufficiency was considered a major complication.

All statistical analyses were performed with SPSS (version 17.0; Chicago, IL). Variables are presented as absolute numbers and percentage or median values and ranges. Statistical comparisons of groups were performed with nonparametric tests, using the MannWhitney $U$ test for continuous variables and the $\chi^{2}$ or Fisher exact test for categorical data. Statistical significance was determined at $P<0.05$. All tests were 2 -sided.

\section{RESULTS}

During the study period, of the 1305 hepatectomies, a total of 485 noncirrhotic patients $(37.2 \%)$ underwent resection of 3 or more liver segments. In all, 243 patients met the inclusion criteria and were enrolled in this study whereas the remaining 242 were excluded because of unavailable anthropometric data $(n=135)$ or CT scans $(\mathrm{n}=107)$. There were 130 men and 113 women, with a median age of 63 years. The demographic, anthropometric, and clinical data of the study population are given in Table 1. More than half of the patients $(51.9 \% ; \mathrm{n}=126)$ had a normal weight for their height [body mass index $\left.(\mathrm{BMI})=16.6-24.9 \mathrm{~kg} / \mathrm{m}^{2}\right]$, whereas the remaining patients were overweight $\left(\mathrm{BMI}=25-29.9 \mathrm{~kg} / \mathrm{m}^{2}\right)(36.6 \%$; $\mathrm{n}=89)$ or obese $\left(\mathrm{BMI} \geq 30-49.8 \mathrm{~kg} / \mathrm{m}^{2}\right)(11.5 \% ; \mathrm{n}=28)$. Indications for resection were in most cases metastatic diseases $(56.8 \%)$ from colorectal $(n=124)$ or noncolorectal $(n=14)$ cancers, followed by primary liver tumors $(38.3 \%)$ of either hepatocellular or biliary origin (hepatocellular carcinoma: $\mathrm{n}=21$; hilar cholangiocarcinoma: $\mathrm{n}=36$; intrahepatic cholangiocarcinoma: $\mathrm{n}=29$; gallbladder cancer: $n=7)$, and other diseases $(4.9 \% ; n=12)$.

At the time of surgery, resection consisted of major hepatectomies (ie, resection of 3 or 4 Couinaud segments) in 135 patients $(55.6 \%)$ and in extended hepatectomies (ie, resection of 5 or 6 Couinaud segments) in 108 patients (44.4\%). In addition to hepatic resection, 106 patients $(43.6 \%)$ underwent 145 additional major intra-abdominal procedures, including 9 vascular and 46 extrahepatic bile duct resections. Preoperatively, 2 patients $(9.5 \%)$ with hepatocellular carcinoma underwent hepatic arterial chemoembolization whereas 71 patients $(57.2 \%)$ with colorectal liver metastases were treated by systemic chemotherapy [median duration: 8 cycles (range: $4-18)$, with most patients $(51 / 71 ; 71.8 \%)$ having received $>6$ cycles]. One-hundred thirty patients $(53.5 \%)$ experienced 1 or more postoperative complications, which were classified as major, nonfatal morbidities (Dindo grade III-IV) in 48 patients (19.7\%). Postoperative hepatic insufficiency occurred in 28 patients $(11.5 \%)$. There were

\section{TABLE 1. Descriptive Characteristics of the Study Cohort}

\begin{tabular}{|c|c|}
\hline Age, yr & $63(24-81)$ \\
\hline Male:female ratio & $130 / 113$ \\
\hline ASA score $(1 / 2 / 3 / 4)$ & $10 / 95 / 92 / 43 / 3^{*}$ \\
\hline Diabetes mellitus & $29(12.4 \%)^{*}$ \\
\hline Body weight, kg & $70(39-144)$ \\
\hline Body height, $\mathrm{cm}$ & $165(140-190)$ \\
\hline $\mathrm{BMI}, \mathrm{kg} / \mathrm{m}^{2}$ & $24.93(16.6-49.8)$ \\
\hline $\mathrm{BSA}, \mathrm{m}^{2}$ & $1.785(1.287-2.608)$ \\
\hline ICG retention rate at $15 \mathrm{~min}$ & $3.9(01-13.5)^{*}$ \\
\hline \multicolumn{2}{|l|}{ Indications } \\
\hline Primary tumors & 93 \\
\hline Metastatic tumors & 138 \\
\hline Other & 12 \\
\hline \multicolumn{2}{|l|}{ Type of resection } \\
\hline Right hepatectomies & 63 \\
\hline Left hepatectomies & 18 \\
\hline Extended right hepatectomies & 58 \\
\hline Extended left hepatectomies & 24 \\
\hline Right trisectionectomies $\pm \mathrm{S} 1$ & 49 \\
\hline Left trisectionectomies $\pm \mathrm{S} 1$ & 10 \\
\hline Other major hepatectomies & 21 \\
\hline \multicolumn{2}{|l|}{ Additional major procedures } \\
\hline Vascular resection & 9 (PV: 7; HA: $1, \mathrm{PV}+\mathrm{HA}: 1)$ \\
\hline Biliary resection & 46 \\
\hline Lymphadenectomy & 63 \\
\hline Other & 27 \\
\hline \multicolumn{2}{|l|}{ Target FLR safe volume } \\
\hline$>25 \%$ & 112 \\
\hline$>30 \%$ & 131 \\
\hline Preoperative PVO & 44 (PVE: 39; PVL: 5) \\
\hline Before major hepatectomy & $9(6 \%)$ \\
\hline Before extended hepatectomy & $35(32.4 \%)$ \\
\hline
\end{tabular}

Values are expressed as absolute number (\%) for categorical values or median (range) for continuous ones.

${ }^{*}$ Data not available for all patients

ASA indicates American Society of Anesthesiologists; HA: hepatic artery; ICG, indocyanine green; PV: portal vein; PVL, portal vein ligation. 
7 postoperative deaths, for a 90-day perioperative mortality rate of $2.9 \%$. Of note, only one patient died of causes unrelated to the liver. This was a 65-year-old woman who succumbed to an acute distress respiratory syndrome on postoperative day 13 . All other deaths were associated with hepatic insufficiency. Although the other 22 patients who developed hepatic insufficiency did not die, they endured a protracted recovery: median length of stay was 29.5 days (range: 8-90 days) versus 10 days (range: 5-127 days) in patients without postoperative hepatic insufficiency $(P<0.001)$.

Volumetric analysis revealed a significant difference between $\operatorname{mTLV}\left(1375 \mathrm{~cm}^{3}\right.$; range: $\left.658-3081 \mathrm{~cm}^{3}\right)$ and eTLV $\left(1467 \mathrm{~cm}^{3}\right.$; range: $\left.837-2510 \mathrm{~cm}^{3}\right)(P<0.001)$. As shown in Figure 1, compared with the eTLV, the mTLV underestimated the TLV in $60.1 \%$ of the cases, whereas it was similar, within a difference of $\pm 2.5 \%$, in $11.1 \%$ of the cases. In the remaining $28.8 \%$ of the patients, the mTLV was larger than the eTLV. The volumetric difference between the 2 methods was more pronounced in overweight and obese patients in whom it reached statistical significance [mTLV vs eTLV in patients with BMI $<25 \mathrm{~kg} / \mathrm{m}^{2}-1265 \mathrm{~cm}^{3}$ vs $1308 \mathrm{~cm}^{3}(P=0.125)$, with BMI $25-29.9$ $\mathrm{kg} / \mathrm{m}^{2}-1421 \mathrm{~cm}^{3}$ vs $1578 \mathrm{~cm}^{3}(P<0.001)$, and with BMI $\geq 30-49.8$ $\mathrm{kg} / \mathrm{m}^{2}-1644 \mathrm{~cm}^{3}$ vs $1818 \mathrm{~cm}^{3}(P=0.049)$ ] (Fig. 2$)$.

Forty-seven (19.3\%) and $73(30.0 \%)$ patients had an inadequate FLR based on the $\mathrm{mR}_{\text {atio }}$ and the $\mathrm{eR}_{\mathrm{atio}}$, respectively $(P=$ 0.006). Preoperative PVO was used in 44 patients $(18.1 \%)$ more often $-79.5 \%$ of the cases - before an extended hepatectomy. Analysis of the mTLV changes after PVO (calculated as $=[\mathrm{mTLV}$ post-PVO $-\mathrm{mTLV}$ pre-PVO]/mTLV pre-PVO $\times 100)$, revealed that, overall, the mTLV decreased by a median of $-3.7 \%$ (range: $-37.6 \%$ to $+27.8 \%$ ), from $1373 \mathrm{~cm}^{3}$ (range: $852-2600 \mathrm{~cm}^{3}$ ) at baseline to $1350 \mathrm{~cm}^{3}$ (range: $\left.824-2077 \mathrm{~cm}^{3}\right)$ after PVO $(P=0.650)$ (Fig. 3). In particular, $17 \mathrm{pa}-$ tients $(38.6 \%)$ had a reduction of the mTLV of more than $5 \%$ of its initial value, 14 patients $(31.8 \%)$ did not experience any significant mTLV change (the mTLV remained within a range of more than $-5 \%$ to less than $+5 \%)$, and 13 patients $(29.6 \%)$ had an mTLV increase of more than $5 \%$. In patients with an overall reduction of the mTLV after PVO, the risk was of overestimating the $\mathrm{mR}_{\text {atio }}$ and thus underestimating the surgical risk. We observed that in the 17 patients who had an mTLV reduction of more than $5 \%$, the incidence of hepatic insufficiency was higher than in those 27 in whom the mTLV remained stable or increased $(41.2 \%$ vs $18.5 \%)$. This difference, however, was not statistically significant $(P=0.195)$ (Fig. 3 ). In addition, the 2 methods seemed similar in evaluating the degree of hypertrophy, defined as the difference between the $\mathrm{mR}_{\mathrm{atio}}$ or the $\mathrm{R}_{\mathrm{atio}}$ before and after PVO. ${ }^{10}$ In particular, a degree of hypertrophy of more than

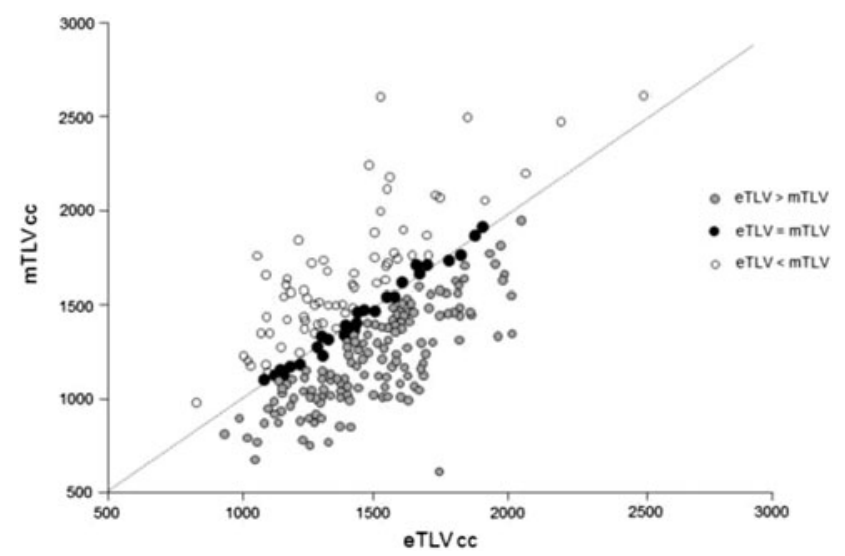

FIGURE 1. Scatter plots showing the correlation between mTLV and eTLV. Dots correspond to individual patients.

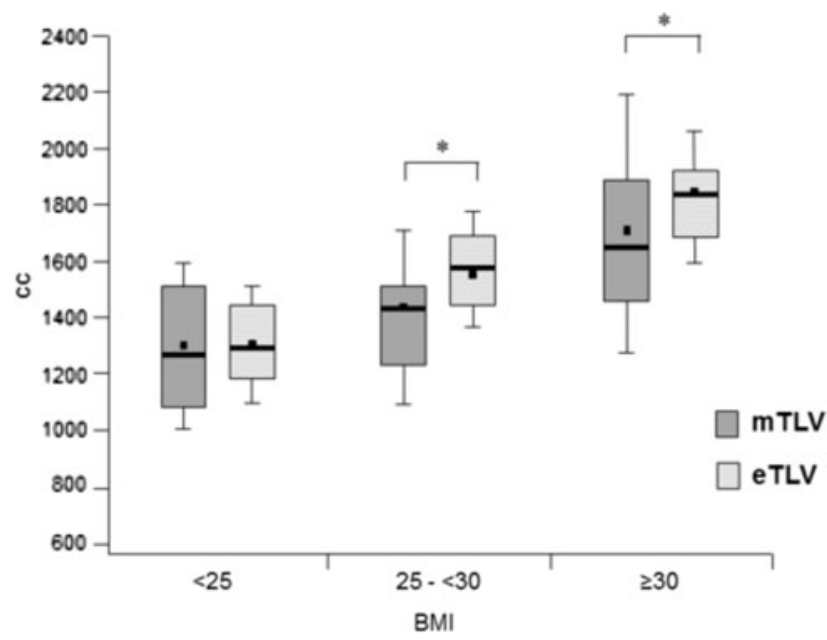

FIGURE 2. Box plots of the MTLV and eTLV stratified by patient size. In each box, the horizontal line is the median; the black square is the mean; the lower and upper borders are the 25th and 75th percentiles, respectively; and the lower and upper whiskers are the standard deviation. *Significant difference $(P<0.05)$.

$5 \%$ was observed in 37 patients $(84.1 \%)$ using the $\mathrm{mR}_{\text {atio }}$ and in 36 patients $(81.8 \%)$ using the $\mathrm{eR}_{\text {atio }}(P=1.000)$.

To further investigate the utility of each method in predicting a safe resection, the study cohort was stratified on the basis of concordance/discordance in indicating the need for PVO. In 207 patients $(85.2 \%)$, the 2 methods were concordant in either indicating $(n=42)$ or not indicating $(\mathrm{n}=165)$ the opportunity to preoperatively stimulate the hypertrophy of the FLR. In these patients, PVO was used in 35 and 3 patients, respectively. In the remaining 36 patients $(14.8 \%)$, the 2 methods were discordant. In particular, in 5 patients, the $\mathrm{mR}_{\text {atio }}$ but not the $\mathrm{eR}_{\text {atio }}$ suggested the need for PVO. In this subgroup, $\mathrm{PVO}$ was used in 2 patients. In contrast, in the other 31 patients, the $\mathrm{R}_{\text {atio }}$ but not the $\mathrm{mR}_{\text {atio }}$ suggested the need for $\mathrm{PVO}$, which was performed in 4 patients. Figure 4 depicts the subgroups stratification. When subgroups outcomes were analyzed, we observed that in patients in whom PVO was not indicated, whatever the method used and thus not performed $(n=162)$, major complications occurred in $16.7 \%$ of the patients, hepatic insufficiency in $4.9 \%$ of the patients, and postoperative death in $0.6 \%$ of the patients. Conversely, in patients who would have been subjected to PVO based on the $\mathrm{eR}_{\text {atio }}$ who instead undergo immediate surgery because of a safe $m R_{\text {atio }}(n=27)$, the incidence of major complications, hepatic insufficiency, and postoperative deaths was higher $(29.6 \%, P=0.108 ; 22.2 \%, P=0.001$; and $3.7 \%$, $P=0.260$, respectively). These results have been summarized in Table 2. It is noteworthy that, among the 31 patients in whom the $\mathrm{eR}_{\text {atio }}$ but not the $\mathrm{mR}_{\mathrm{atio}}$ indicated the necessity of performing a PVO, $18(58.1 \%)$ had a BMI of less than $25 \mathrm{~kg} / \mathrm{m}^{2}$, data that suggest that in this subgroup as well, despite the failure to demonstrate a significant difference in TLVs (Fig. 2), the use of the estimated method (eTLV and $\mathrm{eR}_{\text {atio }}$ ) might be advantageous.

\section{DISCUSSION}

This study indicates that the use of the standardized liver volumetry, ${ }^{8}$ a method based on the estimation of the TLV from the patient's BSA, identifies a not negligible subset of patients in whom the traditional CT-based liver volumetry underestimates the risk of postoperative hepatic insufficiency. 

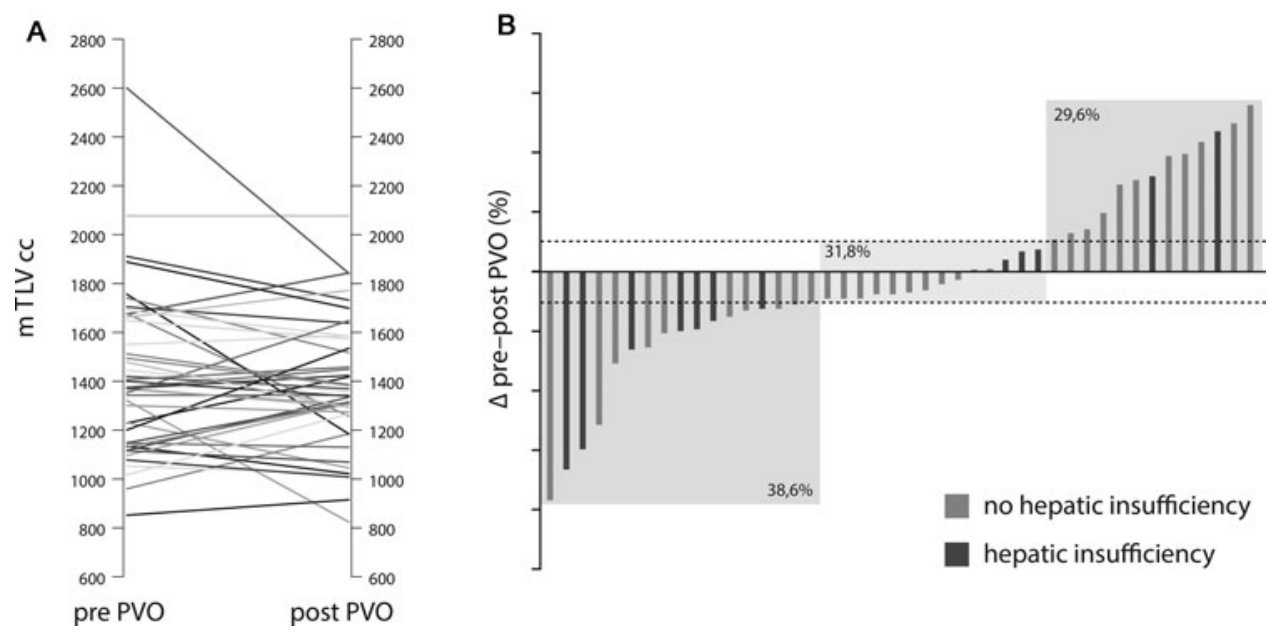

FIGURE 3. Changes of the mTLV after PVO. A, Changes in $\mathrm{cm}^{3}$. B, Waterfall plot of the difference between post- and pre-PVO expressed as a percentage of the initial volume. Each line represent individual patients with (dark gray) or without (light gray) postoperative hepatic insufficiency.

\section{Indication to PVO}
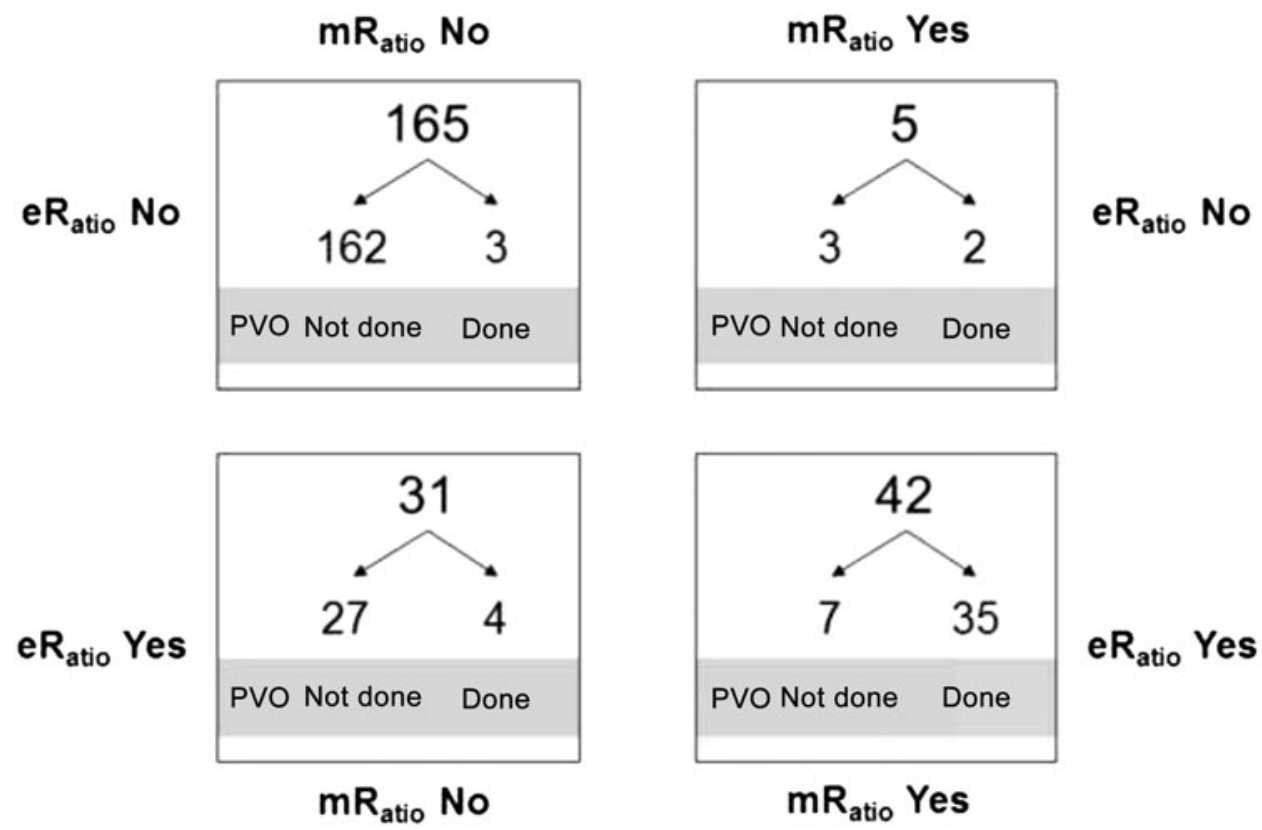

FIGURE 4. Subgroup stratification of the study cohort based on the concordance/discordance of the 2 methods in indicating the need for PVO.

In recent years, major and extended hepatectomies are being used with increasing frequency to achieve complete tumor resection in patients with hepatobiliary malignancies. ${ }^{2,3,7}$ Such extensive resections, however, increase the risk of postoperative complications, hepatic insufficiency, and mortality. ${ }^{4-6}$ On the basis of the association between volume and function of the residual liver, it is being recognized that the preoperative determination of the FLR volume is critical to ensure that sufficient functional parenchyma remains. However, although many have addressed the issue of defining the lower volumetric limits for successful hepatectomies, ${ }^{11-14,22}$ the clinical implications of the method used to measure liver volumes have been rarely investigated. The traditional, most popular method of liver volumetry entails the direct measurements on CT scan sets of the total liver organ and segmental volumes. The FLR volume is then equated to the nontumorous (ie, functional) TLV to calculate a ratio that indicates the percentage of the TLV remaining after resection. The efficacy of this method in discriminating patients at risk for hepatic insufficiency has been repeatedly proved. ${ }^{11,13,14,23}$ The present, large, single-institution series, using validated and strict clinical parameters, including assessment of mortality at postoperative day 90 , reports low major morbidity and mortality rates. Such results further validate this method of liver volumetry. Nevertheless, to measure 
TABLE 2. Baseline Characteristics and Outcome of Patients in Whom Both Methods of Liver Volumetry Did not Show the Need for PVO and of Those in Whom Only the $\mathrm{eR}_{\text {atio }}$ Indicated the Need for PVO

\begin{tabular}{|c|c|c|c|}
\hline & \multicolumn{2}{|c|}{ Indication for PVO } & \multirow[b]{2}{*}{$\boldsymbol{P}$} \\
\hline & $\begin{array}{l}\text { No Need Based } \\
\text { on } \mathrm{mR}_{\text {atio and }} \\
\mathrm{eR}_{\text {atio }}(\mathrm{n}=162)\end{array}$ & $\begin{array}{l}\text { Necessary Only } \\
\text { Based on eR } R_{\text {atio }} \\
\quad(n=27)\end{array}$ & \\
\hline Age, yr & $63(24-81)$ & $65(47-79)$ & NS \\
\hline Male sex & $92(56.8 \%)$ & $13(48.1 \%)$ & NS \\
\hline ASA score $3-4^{*}$ & $97(59.9 \%)$ & $13(48.1 \%)$ & NS \\
\hline Diabetes mellitus* & $23(14.3 \%)$ & $3(11.1 \%)$ & NS \\
\hline $\mathrm{BMI}>25 \mathrm{~kg} / \mathrm{m}^{2}$ & $86(53.1 \%)$ & $12(44.4 \%)$ & NS \\
\hline ICG retention rate at $15 \mathrm{~min}^{*}$ & $3.9(0.1-13.5)$ & $3.1(0.2-11.6)$ & NS \\
\hline Indications & & & NS \\
\hline Primary & $57(35.2 \%)$ & $8(29.6 \%)$ & \\
\hline Metastatic & $96(59.3 \%)$ & $18(66.7 \%)$ & \\
\hline Other & $8(5.5 \%)$ & $1(3.7 \%)$ & \\
\hline Extent of resection & & & NS \\
\hline Major hepatectomy & $106(65.4 \%)$ & $16(59.3 \%)$ & \\
\hline Extended hepatectomy & $56(34.6 \%)$ & $11(40.7 \%)$ & \\
\hline Associated resections & $65(40.1 \%)$ & $9(33.3 \%)$ & \\
\hline Morbidity & $86(53.1 \%)$ & $13(48.1 \%)$ & NS \\
\hline Major complications & $27(16.7 \%)$ & $8(29.6 \%)$ & NS \\
\hline Hepatic insufficiency & $8(4.9 \%)$ & $6(22.2 \%)$ & 0.001 \\
\hline Mortality & $1(0.6 \%)$ & $1(3.7 \%)$ & NS \\
\hline Length of hospital stay, d & $10(5-127)$ & $10(6-90)$ & NS \\
\hline
\end{tabular}

Values are expressed as absolute number (\%) for categorical values or median (range) for continuous ones.

${ }^{*}$ Data not available for all patients.

ASA indicates American Society of Anesthesiologists; ICG, indocyanine green; NS, not significant.

liver and tumor volumes, there are some disadvantages. Although error rates for measuring these volumes on CT scans have decreased to less that $3 \%,{ }^{24}$ in patients with multiple tumors, mathematical errors cumulate because of multiple measurements. In addition, this method relies on the assumption of a homogeneously preserved function of the whole liver. This, however, is not always true. In patients with segmental or sectorial biliary dilation or vascular obstruction, for example, the compromised parenchyma reduces the actual functional liver volume, which does not correspond to the actual TLV. Another shortcoming is that this method does not provide a fixed estimation for TLV before and after PVO; in patients experiencing a marked atrophy of the embolized hemiliver, the use of a smaller post-PVO denominator for calculating the $\mathrm{eR}_{\mathrm{atio}}$ will overestimate FLR hypertrophy. Consistently, in this series, we observed that in $38 \%$ of the patients who had undergone PVO, at the reevaluation preceding resection, the mTLV was actually smaller, with a volume decrease of more than $5 \%$ from the baseline. In such patients, the incidence of hepatic insufficiency was 2 -fold higher than that observed in all other patients. This difference, however, did not reach statistical significance probably because of the small sample size. Besides, the limited number of patients also suggests caution in interpreting these data. The last disadvantage of the traditional method of liver volumetry is its possible inadequacy in patients selected for repeat hepatectomy because liver regeneration after the first hepatectomy allows no more than $85 \%$ of the original liver volume to be recovered. ${ }^{25}$

An alternative method, defined as standardized liver volumetry, estimates the ideal TLV, using a formula developed on a large series of patients undergoing $\mathrm{CT}$ for conditions unrelated to the hepatobiliary system and based on the correlation existing between liver size and BSA. ${ }^{9}$ The BSA is widely used as the biometric unit for normalizing physiological parameters such as cardiac output, left ventricular mass, or renal clearance and for determining the appropriate chemotherapy drugs dosage in individuals of different body size. Estimating the TLV with this BSA-based formula, which has been found to be the most precise and unbiased equation among 12 existing models ${ }^{26}$ corrects the actual liver volume to the individual patient's size and provides a validated, individualized estimate of that patient's postresection liver function. In addition, this method overcomes most of the shortcomings of the traditional method.

Whether remnant liver volume should be evaluated as a ratio of FLR to mTLV or eTLV remains unknown. In this study, we sought to determine which method of liver volumetry is more relevant in assessing the minimum, safe FLR volume after major or extended hepatectomy. We found that the $\mathrm{e}_{\text {atio }}$ allowed for better identification of a subset of patients at risk for hepatic insufficiency. In particular, in approximately $11 \%$ of our patients, we observed a discrepancy between the $\mathrm{eR}_{\text {atio }}$ and the $\mathrm{mR}_{\text {atio }}$, with the $\mathrm{mR}_{\text {atio }}$ falsely predicting a safe resection. Interestingly, although significant differences in TLVs were observed only in overweight or obese patients, differences in corresponding ratios were uniformly distributed across the spectrum of different body sizes, suggesting that the standardized liver volumetry ( $\mathrm{eTLV}$ and $\mathrm{R}_{\mathrm{atio}}$ ) might be advantageous in all patients. Yet, if the $\mathrm{eR}_{\text {atio }}$ were routinely used, we would have 1.5 -fold increased use of PVO. Robust evidence indicates that PVO is highly effective in increasing the FLR mass preoperatively and that this hypertrophy significantly reduces the risk of postoperative hepatic insufficiency. But PVO causes adverse events in approximately $10 \%$ of the patients, ${ }^{10,27}$ and tumor progression after the procedure may occur. ${ }^{28-30}$ Therefore, the clinical implication of expanding the number of candidates to PVO will have to be carefully evaluated in the future studies. In particular, future studies should evaluate whether the reduction of hepatic insufficiency will be counterbalanced by PVE-related adverse events.

This study suffers from the intrinsic limitations of any retrospective analysis. In addition, because we analyzed a selected population, having excluded some patients might infer a risk for bias. However, we believe that meeting or not meeting our selection criteria was just a matter of chance and that the risk for an unbiased selection was minimum. Finally, the composition of our study cohort comprising disparate tumor types may have conditioned heterogeneous outcomes because different cancer patients may be at a different risk. Nonetheless, liver volumetry is a method established to assess the risk of major or extended hepatectomy whatever the indication; different threshold volumes for safe resection address the issue of different levels of risk in different patients.

\section{CONCLUSIONS}

We showed that the use of the standardized volumetry identifies a subset of patients in whom the traditional method of liver volumetry significantly underestimates the risk of hepatic insufficiency. Because of its numerous advantages, this method of liver volumetry should be more widely adopted.

\section{REFERENCES}

1. Vauthey JN, Pawlik TM, Abdalla EK, et al. Is extended hepatectomy for hepatobiliary malignancy justified? Ann Surg. 2004;239:722-730.

2. Neuhaus P, Thelen A, Jonas S, et al. Oncological superiority of hilar en bloc resection for the treatment of hilar cholangiocarcinoma. Ann Surg Oncol. 2012;19:1602-1608.

3. Natsume S, Ebata T, Yokoyama Y, et al. Clinical significance of left trisectionectomy for perihilar cholangiocarcinoma: an appraisal and comparison with left hepatectomy. Ann Surg. 2012;255:754-762.

4. Mullen JT, Ribero D, Reddy SK, et al. Hepatic insufficiency and mortality in 1,059 noncirrhotic patients undergoing major hepatectomy. J Am Coll Surg. 2007;204:854-862; discussion 862-854. 
5. Jarnagin WR, Gonen M, Fong Y, et al. Improvement in perioperative outcome after hepatic resection: analysis of 1,803 consecutive cases over the past decade. Ann Surg. 2002;236:397-406.

6. Halazun KJ, Al-Mukhtar A, Aldouri A, et al. Right hepatic trisectionectomy for hepatobiliary diseases: results and an appraisal of its current role. Ann Surg. 2007;246:1065-1074.

7. Kishi Y, Abdalla EK, Chun YS, et al. Three hundred and one consecutive extended right hepatectomies evaluation of outcome based on systematic liver volumetry. Ann Surg. 2009;250:540-548.

8. Vauthey JN, Chaoui A, Do KA, et al. Standardized measurement of the future liver remnant prior to extended liver resection: methodology and clinical associations. Surgery. 2000;127:512-519.

9. Vauthey JN, Abdalla EK, Doherty DA, et al. Body surface area and body weight predict total liver volume in Western adults. Liver Transpl. 2002;8: 233-240.

10. Ribero D, Abdalla EK, Madoff DC, et al. Portal vein embolization before major hepatectomy and its effects on regeneration, resectability and outcome. $\mathrm{Br} J$ Surg. 2007;94:1386-1394.

11. Ferrero A, Vigano L, Polastri R, et al. Postoperative liver dysfunction and future remnant liver: where is the limit? Results of a prospective study. World J Surg. 2007;31:1643-1651.

12. Abdalla EK, Barnett CC, Doherty D, et al. Extended hepatectomy in patients with hepatobiliary malignancies with and without preoperative portal vein embolization. Arch Surg. 2002;137:675-680; discussion 680-681.

13. Schindl MJ, Redhead DN, Fearon KC, et al. The value of residual liver volume as a predictor of hepatic dysfunction and infection after major liver resection. Gut. 2005;54:289-296.

14. Shoup M, Gonen M, D'Angelica M, et al. Volumetric analysis predicts hepatic dysfunction in patients undergoing major liver resection. $J$ Gastrointest Surg. 2003;7:325-330.

15. Macera A, Lario C, Petracchini M, et al. Staging of colorectal liver metastases after preoperative chemotherapy. Diffusion-weighted imaging in combination with Gd-EOB-DTPA MRI sequences increases sensitivity and diagnostic accuracy. Eur Radiol. 2013;23:739-747.

16. Ribero D, Amisano M, Lo Tesoriere R, et al. Additional resection of an intraoperative margin-positive proximal bile duct improves survival in patients with hilar cholangiocarcinoma. Ann Surg. 2011;254:776-781; discussion 781-783.

17. Capussotti L, Muratore A, Baracchi F, et al. Portal vein ligation as an efficient method of increasing the future liver remnant volume in the surgical treatment of colorectal metastases. Arch Surg. 2008;143:978-982.

18. Capussotti L, Muratore A, Ferrero A, et al. Extension of right portal vein embolization to segment IV portal branches. Arch Surg. 2005;140:1100-1103.

19. Mosteller RD. Simplified calculation of body surface area. $N$ Engl J Med. 1987;317:1098.

20. Dindo D, Demartines N, Clavien PA. Classification of surgical complications: a new proposal with evaluation in a cohort of 6336 patients and results of a survey. Ann Surg. 2004;240:205-213.

21. Rahbari NN, Garden OJ, Padbury R, et al. Posthepatectomy liver failure: a definition and grading by the International Study Group of Liver Surgery (ISGLS). Surgery. 2011;149:713-724.

22. Clavien PA, Petrowsky H, DeOliveira ML, et al. Strategies for safer liver surgery and partial liver transplantation. N Engl J Med. 2007;356:1545-1559.

23. Farges O, Belghiti J, Kianmanesh R, et al. Portal vein embolization before right hepatectomy: prospective clinical trial. Ann Surg. 2003;237:208-217.

24. Tongyoo A, Pomfret EA, Pomposelli JJ. Accurate estimation of living donor right hemi-liver volume from portal vein diameter measurement and standard liver volume calculation. Am J Transplant. 2012;12:1229-1239.

25. Nadalin S, Testa G, Malago M, et al. Volumetric and functional recovery of the liver after right hepatectomy for living donation. Liver Transpl. 2004;10:10241029.

26. Johnson TN, Tucker GT, Tanner MS, et al. Changes in liver volume from birth to adulthood: a meta-analysis. Liver Transpl. 2005;11:1481-1493.

27. Di Stefano DR, de Baere T, Denys A, et al. Preoperative percutaneous portal vein embolization: evaluation of adverse events in 188 patients. Radiology. 2005;234:625-630.

28. Kokudo N, Tada K, Seki M, et al. Proliferative activity of intrahepatic colorectal metastases after preoperative hemihepatic portal vein embolization. Hepatology. 2001;34:267-272.

29. Muratore A, Zimmitti G, Ribero D, et al. Chemotherapy between the first and second stages of a two-stage hepatectomy for colorectal liver metastases: should we routinely recommend it? Ann Surg Oncol. 2012;19:1310-1315.

30. Hoekstra LT, van Lienden KP, Doets A, et al. Tumor progression after preoperative portal vein embolization. Ann Surg. 2012;256:812-818.

\section{DISCUSSANTS}

\section{P. Neuhaus (Germany):}

That was an interesting talk. Let me say first that until today I found it difficult to understand the usefulness of an estimated liver volume based on BSA in comparison to CT-based volumetric calculation, which is, in fact, used in most hepatobiliary centers. Liver size may vary considerably, and it is unclear why an estimation based on BSA should be better than a seemingly more precise measurement. A crucial question is assessment of liver function and prediction of liver regeneration. With regard to liver function, the LiMAx test, which will be soon available, is much superior to indocyanine green (ICG) and other tests. However, for prediction of the regenerative capacity of the FLR, we still do not have good tools, although we know many things that might impair liver regeneration, for example, liver pathologies such as sclerosis, age of the patient, and concomitant sepsis. Is it possible that your calculation of TLV in critically ill patients with large fatty livers gives a considerably lower estimated volume and a very low FLR volume? This would mean that patients with oversized and poorly functioning livers might be excluded from resection. Another point is that besides your main outcome parameters such as mortality, hepatic insufficiency, and major surgical complications, there are other factors such as extent of resection, blood loss, postoperative liver function, age, biliary procedures, and so on, that influence the outcome. From your article, I could see that you did a univariate analysis, but do you have any data using multivariate analysis? I thank you again for the presentation and the privilege to comment.

\section{Response From D. Ribero (Turin, Italy):}

Professor Neuhaus, thank you very much for your thoughtful comments. I will just go quickly through all of your questions. We believe that using the estimated liver volume adds something in a small group of patients, approximately $11 \%$ of the candidates for major or extended liver resection. In this subgroup of patients, we found $22 \%$ liver insufficiency. Thus, we need to move forward because we would like to avoid postoperative liver insufficiency.

It is difficult to exactly explain why the estimated liver volume can be better than the measured liver volume. The estimation is based on a formula that was derived from a large population of healthy patients. On the basis of this, we could calculate the ideal liver volume for an individual patient. We know that each patient may have an enlarged liver or a shrunken liver because not all patients are equal. With the estimated volume, we try to standardize the volume of the liver to the patient size. We know that liver function is extremely important in determining the outcome; however, there are many ways to assess the function of the liver. The LiMAx test is certainly an important test. We usually use the ICG test, which is well recognized. The liver function in our patients was excellent, with a retention rate of approximately 3.9. So, in patients who might be candidates for a major or extended hepatectomy, beside the function, we need to find something that helps define the surgical risk, and liver volume has been shown to help in this risk assessment.

With regard to your last comment, obviously in surgery, we have many components that might contribute to development of liver insufficiency. However, before resection, we need a tool that should be valuable in all patients, for any type of operation, and for any kind of indication. Thank you.

\section{DISCUSSANTS}

\section{F. Pruvot (Lille, France):}

Congratulations on your article, one comment and one question. Do you not think that comparison of measured and estimated 
TLV is an old story? We showed that the liver remnant to body weight ratio is a more accurate method to calculate the risk of liver resection. We published this in 2007 and presented this review in Helsinki in 2011. So, please could you recalculate your data with ratio to body weight as a third method and send us the results; I am very interested.

My question is that you showed a particular subgroup with increased morbidity, with decrease in TLV after portal vein embolization. Was it because of atrophy of embolized liver or insufficient hypertrophy of the remnant?

\section{Response From D. Ribero (Turin, Italy):}

I agree with your comment on FLR to body weight. We also published before on this topic, and what we have shown is that the FLR to body weight was exactly the same as using FLR to the estimated TLV. The problem in using the FLR to body weight is that those who do not perform liver transplantation are not familiar with the results. Most of the established cutoff points we showed are in percentages, so people are more used to have percentages.

We do not know the reason for the liver shrinkage; we did not look at each single-segment volume; however, the problem remains in those patients in whom looking at the CT scan we had a significant shrinkage of the right lobe rather than hypertrophy of the FLR. This may be a problem because if we decrease the denominator, we might overestimate the liver growth.

\section{DISCUSSANTS}

\section{T. van Gulik (Amsterdam, The Netherlands):}

Thank you, I enjoyed your article. I agree with you that the estimated liver volume is attractive because it might correct the miscalculations in, for instance, vascular spaces and the tumor lobe. But it is based on a normal population with normal livers and that is why you excluded all the cirrhotic livers because you cannot apply it on cirrhotic livers but you do apply it on patients who had combined resections because of bile duct cancer. Those are the patients you should also have included because they are not normal livers; they are cholestatic livers or postcholestatic livers and therefore must skew your results. So, I wonder how homogenous is the patient population in order that you can use the estimated volume? Another point is that it makes a difference if you use the formula in Asian population or in European population. My final question is that you have done an ICG clearance test in all patients but you have not used it in your analysis in terms of determining patients who have an insufficient liver functional reserve. Why is that?

\section{Response From D. Ribero (Turin, Italy):}

I will start with the last one. We used the ICG test in all nonjaundiced patients when the bilirubin level was below $2 \mathrm{mg} / \mathrm{dL}$, and if you look at our results, the upper ICG limit at 15 minutes was 13. There are data from the East that showed us that in cirrhotic patients, we could also do major hepatectomy in patients with ICG limits up to 14; so, we think that liver function was good. I do not completely agree with you about patients with jaundice; of course, if you measure the volume in a jaundiced patient, this might be enlarged because of the dilation of the biliary tree. But this is a patient with basically a normal liver, or at least noncirrhotic. So with the formula, we estimate the theoretical volume that the patient would require; it does not matter if now the patient has an enlarged liver because of the jaundice, which might have lasted for the past couple of weeks. On the contrary, I agree with you that we need to look more closely at cirrhotic patients.

\section{DISCUSSANTS}

\section{C.H.C. Dejong (Maastricht, The Netherlands):}

You have a median BMI of 25, so half are above and half are below this BMI. This is another confounding factor because there are patients who are obese and yet sarcopenic. You can measure the volume of the muscle at the L3 level, and they may regenerate less and they may have less functional recovery. I think you should be invited to look more closely at the level of sarcopenia in these patients, even the obese patients.

\section{C.M. Lo (Hong Kong, PR China):}

I like your conclusion because I have used estimated liver volume, but I do not think your data can support the conclusion. Your data are basically supported by the 27 patients in whom the estimated TLV would indicate a need for portal vein embolization and you end up with 6 having liver insufficiency. But your definition of hepatic insufficiency is only based on jaundice, where you have patients who have bile duct resection as well. There are many reasons that patients can have jaundice after hepatic resection, particularly when you do bile duct resection and other procedures, infection, etc. So, basing your conclusion on 6 of 27 patients with hepatic insufficiency is very flimsy, and I think you may have to reconsider your definition for liver insufficiency and look into these 6 patients.

\section{Response From D. Ribero (Turin, Italy):}

The problem of the definition of liver insufficiency is always a major issue and what we did was just to use the established criteria that have been published after a consensus of expert liver surgeons; using an established definition of liver insufficiency is the only way we have to compare studies. But still, this definition remains a problem. About the cachexia, we do not operate cachectic patients. 